\title{
ELABORACION DEL INVENTARIO DE INTERESES VOCACIONALES PARA CARRERAS TÉCNICAS Y DE OFICIO
}

\author{
Luis A. Vicuña Peri
}

La presente investigación tuvo como fin elaborar el Inventario de Intereses hacia Carreras Técnicas y Oficios, para lo cual se tuvo en cuenta las escuelas, centros e institutos que ofrecen capacitación tecnológica y artesanal, para reconocer áreas tecnológicas que fueran útiles para el procedimiento de validez de contenido, en el que actuaran como expertos técnicos calificados con experiencia no menor de cinco años, por psicólogos especialistas en consejería y asesoría vocacional. A través de este procedimiento el inventario demostró su validez, así como por el método de constructos, en el que se verifica que las áreas contempladas sean independientes y que puedan ser reagrupadas en cuatro áreas mayores hecho que facilita la aplicación porque nos permite reducir el numero de ítemes del inventario.

Respecto a la confiabilidad, se usaron tanto el método de la consistencia interna como el método de test retest, encontrándose que el inventario mide con mínimos márgenes de error.

Los baremos elaborados son de puntaje $\mathrm{T}$, para varones y mujeres al encontrar que en 10 de las 15 áreas el sexo plantea diferencias significativas en los valores promedio.

PALABRAS CLAVE. Interés Intrínseco, Interés extrínseco, carrera técnica, validez, confiabilidad.

The present investigation was oriented to elaborates you the Inventory of Interests toward Technical Careers and of Position, for which was explored the schools, centers and institutes that offer craft and technological training, with it 1 am grouped technological areas that served to plan the content validity procedure performed by technicians qualified with not smaller experience of five years, and by psychologists specialists in consejería and vocational advising, by this Procedure the inventory showed their validity likewise by the method of construct, where is verified that the areas contemplated plows independent and that dog be regrouped in four greater areas, fact that facilitates the application because permits us to reduces the 1 number of ítemes of the inventory.

Regarding the dependability so much by the method of the internal consistency ace by the method of the test retest. the inventory measures with most minimum margins of error.

The scales elaborates you it plows of puntaje $\mathrm{T}$, for bad and women upon finding that in 10 of the 15 areas the sex presents differentiates significant in the values average.

KEY WORDS: Intrinsic interest, extrinsic Interest, technical career, validity, dependability. 


\section{INTRODUCCION}

Los estudios efectuados en 1984 por el autor dejaron entrever la necesidad de contar con instrumentos de medición psicológica adecuados a la forma en que la actividad económica y los puestos de trabajo, sean estos dependientes o independientes, se vinculan interactivamente. Con tal fin se elaboró el inventario de intereses vocacionales para profesiones universitarias, instrumento que ha sido estandarizado en Lima, Huancayo y Trujillo. No obstante, en los últimos diez años, cuando orientamos vocacionalmente a grupos de varones y mujeres, pertenecientes a centros educativos de gestión estatal y privada encontramos que las profesiones universitarias obtienen cada vez puntuaciones relativamente bajas, lo que estaría indicando que nuestros jóvenes vienen desarrollando intereses hacia otras áreas de" la actividad humana, que posiblemente se acomoden hacia carreras cortas de nivel tecnológico y también hacia ocupaciones de oficio muy artesanal. Para corroborar los resultados arriba mencionados, el año 2000 el autor de la presente investigación realizó una encuesta aplicada a cinco centros educativos de la ciudad de Lima, tres de los cuales fueron de gestión estatal y dos de gestión privada, en la que encontramos que cerca de un $25 \%$ de jóvenes anotaban como primera opción de vida laboral ocupaciones tales como: cocina (chef), panadería, técnico dental, ensamblaje de computadoras, rehabilitación y fisioterapia, así como modas, diseño. Cosmetología entre otras, estos datos en sí mismos otorgan especial' Importancia al presente tema de estudio, más aún cuando en la investigación para el Instituto de Investigaciones Psicológicas. Vicuña (2000), hemos encontrado que el 11\% de los ingresantes a la Universidad en el concurso de admisión del año 2000 manifiestan su deseo de trasladarse a otras especialidad, que se caracterice por ser práctica, breve y rentable. Como se puede observar estos requerimientos no son para profesiones universitarias, sino para carreras cortar donde la habilidad y el talento se combinan para ofrecer servicios, organizar negocios y también emprender la configuración de micro empresas de producción.

La inquietud actual de los jóvenes debe ser detectada a tiempo para la orientación o reecanalización de sus habilidades, personalidad, intereses y motivaciones hacia planes de vida donde conjuguen sus aspiraciones con sus aptitudes dentro del contexto socio económico en el que se desenvuelven, con el propósito de reducir al máximo, las deserciones y frustraciones académicas, así como la pérdida de tiempo con el alto costo tanto para el estudiante como para la sociedad.

Por otro lado atendiendo a la OIT, que en su informe anual de 1998, da cuenta que uno de los principales problemas en el mundo es el desempleo y el subempleo y, que su solución mas allá de la planificación económica, requiere de una educación que vaya de la mano con las nuevas demandas que el mundo de hoy está generando, ya que el avance tecnológico va desapareciendo de la oferta laboral a ocupaciones tradicionales, por ejemplo los auxiliares de los cajeros en las agencias bancarias, los cargadores o estibadores de los puestos marítimos, los auxiliares de secretarias, quienes se fueron junto a otros definitivamente, sustituidos por las máquinas de tecnología de punta, generando la pérdida de miles de puestos de trabajo; sin embargo este avance no es totalmente negativo, porque a su vez están generando nuevas necesidades de mano de obra, cada vez con mayor capacitación. Surgen nuevos puestos, por ende con nuevos perfiles, donde la enseñanza y la orientación vocacional sobre las ocupaciones tradicionales están perdiendo piso aceleradamente, demandando la revisión de los enfoques y que aceptemos el reto de las nuevas demandas laborales, para optimizar el proceso orientador. 
En lo último se justifica el presente estudio, que aborda precisamente el problema de la orientación vocacional y ocupacional, en un ámbito sociofamiliar actual que hace dificil la decisión hacia un plan de vida ad-hoc con las aptitudes, intereses, demanda social laboral y las percepciones existentes hacia el status social de las ocupaciones técnicas y de oficio.

Creemos que la claridad que el interesado tenga de sí mismo, le hará más objetiva y plástica la toma de decisiones hacia un plan de vida; donde la motivación intrínseca actuará como un impulsor que mantengan las pertinentes hacia el logro de la meta deseada conduciendo a la autorrealización.

Para contribuir con la claridad hacia sí mismo, el producto de la presente investigación consiste en proporcionar a la comunidad educativa en general y en particular para la orden profesional de psicólogos un inventario válido y con fiable, para diagnosticar los intereses hacia carreras técnicas y de oficio.

Para cumplir con la tarea propuesta, tendremos en primer lugar, luego de revisar la gama de capacitaciones tecnológicas que ofrecen los diferentes centros de capacitación, agruparlas por áreas, para lo cual se tendrá en cuenta la afinidad de roles, funciones y exigencias cognitivos conductuales que demandan cada una de las diferentes áreas a medirse, esto exige la demostración de hecho que las áreas tecnológicas pre establecidas resulten válidas.

En segundo lugar veremos que cada una de las áreas de interés tecnológico midan con el menor riesgo de error.

Efectuado los dos pasos previos, se explorará si se justifica la elaboración de normas para categorizar los intereses para cada área según el sexo, la decisión se tomará contrastando los resultados promedios entre los varones y mujeres examinados.

La elaboración del inventario tendrá en cuenta el enfoque de los rasgos psicológicos, enfoque que parte de la suposición que los puestos laborales exigen de un perfil psicológico específico donde los intereses tiene un papel importante que deben combinar positivamente con las aptitudes diferenciales y una caractereología permisible y facilitadora para los roles y funciones de un determinado puesto. Rivas (1988) Este enfoque parte de los siguientes supuestos que enfatiza la teoría de las diferencias individuales:

- Cada sujeto puede ser caracterizado según un conjunto de rasgos que se miden con fiabilidad y validez.

- Los puestos de trabajo exigen que los trabajadores posean ciertos rasgos y en el nivel necesario para el buen desempeño.

- La elección de una ocupación es un proceso en el que puede lograrse la relación de ajuste más adecuada.

La mayor probabilidad de éxito (productividad y satisfacción) se consigue cuando mejor es la adecuación entre las características individuales y las exigencias del trabajo.

Dentro de este enfoque, nuestro trabajo enfatiza en la medición de los intereses hacia un determinado campo ocupacional tecnológico, que nos permita explorar el grado de identificación, con los roles y funciones. De esta manera esperamos que los examinados luego de emitir sus respuestas selectivas entre pares de roles y funciones accedan al conocimiento hacia qué área tecnológica presenta mayor interese.

Consideramos que el interés es un componente motivacional que actúa como el motivo 
que sostiene a largo plazo las conductas de los individuos hasta su consecución, cuando es primaria esta cesa una vez alcanzada, y volverá a impulsar cuando sea notorio para el individuo su ausencia por ejemplo el interés por el alimento, el agua, la sexualidad y otras de naturaleza similar. Cuando el interés es secundario, actúa de manera similar al interés primario, en lo que respecta al impulso o fuerza, para conseguir la meta, pero a diferencia del primario, en el interés secundario el motivo aún cuando fuese extrínseco como por ejemplo tener un automóvil, o una casa propia, el interés no se agota con tales logros, porque ello mismo impulsa hacia nuevas metas asociadas, por ejemplo, conseguido el automóvil, ahora es muy probable que se anhele un carro más potente o más caro o más confortable, en fin al parecer no hay límite para la voluntad humana que se inspira en lo posible, reconociendo sus limitaciones, algo similar ocurre en el ejemplo de la casa propia conseguida, tendrá el impulso por amoblarla, luego tal vez quiera una más grande o en otra zona. Por otro lado, cuando el interés secundario es intrínseco, el motivo no está fuera, sino que está en el mismo sujeto, quien responde a las preguntas ¿qué quiero lograr?, ¿qué quiero hacer de mi persona?, ¿cómo me veré haciendo tal o cual actividad?, ¿Dónde puedo alcanzar lo que quiero?; como podemos ver, las respuestas están dirigidas hacia logros que quedan en uno mismo, no se materializan como cuando se compra un automóvil o una casa propia. La materialización en este caso, es la satisfacción de haber alcanzado una meta la que a su vez inicia un camino, es decir una carrera hacia metas que se van sucediendo y que son de logro individual, nos referimos si una persona quiere ser un ebanista, conseguirlo es haber utilizado sus repertorios conductuales de coordinaciones motoras finas, más las habilidades estéticas que deben plasmarse en productos tangibles, la meta obviamente no está en el mueble, la meta ha estado en conseguir la destreza para transformar materias primas en artefactos de uso conocido, y también conseguida la meta, ahora no estará satisfecho con el rótulo de ebanista, apuntará hacia la configuración empresarial dentro de lo suyo, aquí el concepto de carrera, además de significar secuencia de puestos ocupados por una persona concreta a través de $\mathrm{Su}$ vida pre-profesional, profesional y post ocupacional, incluyendo distintos roles laborales y sociales asociados que van desde un estudiante, profesionales activos a jubilados Súper (1983), que se ajusta mejor para las profesiones universitarias, para nosotros incorpora a las ocupaciones denominadas de mando medio o técnicas, que también califican en función a la trayectoria ocupacional.

El interés por carreras técnicas, actúa como un impulsor psicológico que dirige la atención, y organiza la vida en pro el desarrollo de conocimientos y destrezas de carácter práctico, las que se forman a partir de aptitudes que predisponen al individuo para realizar con relativa facilidad, eficiencia y eficacia tareas específicas. La retroinformación conductual, fortalece al interés, con ello permite que las personas mantengan prolongados estados de entrenamiento, dedicación al estudio o la práctica, sacrificando horas de descanso o tal vez de sana diversión. Los intereses incluyen el apego por roles y las funciones específicas que definen los puestos de trabajo, dependientes o liberales. Vinculados con la producción que va desde lo artesanal hasta la gran industria, pasando por la mediana y pequeña empresa de producción y servicios; sin embargo a lo largo de la vida del individuo, su entorno social inmediato, tal como su familia, luego la escuela y simultáneamente sus amigos, presionan siguiendo las políticas del momento, de forma tal que el individuo en vez de encontrar un cauce libre y facilitador para su autorrealización, se encuentra con un cauce plagado de tabiques, los que dificultan la libre expresión de sus tendencias naturales para asumir y dirigir su atención hacia campos ocupacionales emergentes, pero que en el común .de los casos no guardan relación con los intereses básicos del individuo. Esta situación genera duda y desorientación sobre todo al 
adolescente, quien debe asumir un rol protagónico en la delimitación de su futuro. Este penoso estado de confusión debe ser clarificado, permitiendo que el joven pueda reencontrase.

Con este propósito el presente trabajo tiene como objetivo principal elaborar un inventario que nos permita medir los intereses hacia carreras técnicas y de oficio de mayor demanda en el mercado laboral, siendo posible la identificación de áreas de preferencia con la posibilidad de obtener el perfil ocupacional técnico y de oficio del examinado.

Trabajamos con las siguientes hipótesis:

H1. El inventario tendrá una confiabilidad mayor de 0.80 por el método de la consistencia interna, para cada una de sus áreas de interés.

H2. El inventario tendrá una confiabilidad mayor de 0.80 por el método del test- retest, para cada área de interés.

H3. El inventario tiene validez de contenido, de constructo por análisis factorial y por el método de discriminación por especialidad técnico o de oficio.

H4. El inventario discrimina los promedios de los indicadores de interés, en función al sexo, siendo necesaria la elaboración de normas específicas para varones y mujeres.

\section{METODOLOGIA}

La presente investigación es psicométrica con un diseño descriptivo correlacional, y comparativa de corte transversal, teniendo como propósito elaborar el inventario de intereses hacia carreras técnicas y de oficio, en jóvenes desde los 15 hasta los 22 años, teniendo en cuenta además de la edad el género como variables que pueden afectar en alguna medida y dirección los intereses hacia carreras técnicas y de oficio.

POBLACIÓN y MUESTRA.- El universo está constituido por 1'289,364 jóvenes comprendidos entre los 15 hasta los 24 años de edad según el censo de 1993 cuya distribución esta descrita en la tabla 1.

Para determinar la muestra hemos aplicado el procedimiento de muestreo probabilístico estratificado, para un margen de error del 0.05 y un nivel de confianza del $0.95 \%$ utilizando como estimador la misma probabilidad de elección utilizando la fórmula de Cochran, requiriendo un tamaño de muestra igual a 400 jóvenes, que serán distribuidos proporcional-mente a cada estrato y la elección final será aleatoria.

\section{ELABORACION DEL INVENTARIO DE INTERESES PARA CARRERAS TECNICAS. (INCATEC-2001).}

Para lograr nuestro propósito hemos desarrollado los siguientes pasos:

a) Acopio de información, con el propósito de identificar el número tanto de centros de capacitación tecnológica, artesanal y las especialidades que imparten, este paso nos llevo tres meses registrando avisos periodísticos, avisos clasificados, se acudieron a los centros 
para recoger información complementaria y como producto se obtuvo ciento nueve centros distribuidos tal como se presenta en el cuadro $\mathrm{N}^{\circ} 2$ :

b) Sumando los totales del cuadro anterior tiene 109 centros de capacitación tecnológicos, los que ofrecen bajo diferentes denominaciones entrenamientos similares respecto a las funciones y servicios que delimitan una determinada especialidad, del análisis efectuado consideramos que pueden agruparse en quince áreas, y que a su vez cada una exige de habilidades e intereses específicos, el perfil de habilidades e intereses se obtuvo entrevistando y cuando era posible mediante conversatorio en pequeños grupos con técnicos que trabajan en cada una de las especialidades por un tiempo no menor de cinco años. Con ambos datos, áreas y habilidades más intereses se elaboró la tabla de especificaciones cuyo contenido presentamos a continuación.

AREA I. Electrónica y Electricidad Habilidades Sensoperceptuales: visión cromática que facilite la percepción de funciones.

Motivación: interés por aprender funciones, manejo de herramientas.

Técnico científico: causalidad empírica de naturaleza física.

Pragmatismo: Meticulosidad pragmática. Motriz: motricidad fina.

Cálculo: de descargas electrofisicomecánicas.

Posibles ocupaciones: Técnico en hardware, electrónica y electricidad doméstica e industrial refrigeración y aire acondicionado.

\section{AREA II. Seguridad}

Habilidades Sensoperceptuales: agudeza visual y auditiva.

Motivación: interés por proteger, custodiar y salvaguardar.

Técnico científico: Manejo estratégico de la posición y lugar.

Pragmatismo: exponer la vida por el bien de otros.

Motriz: reacción refleja rápida y sostenida.

Cálculo: magnitud del riesgo y peligro.

Posibles ocupaciones: Agente de seguridad, Salvavidas, Investigador.

AREA III. Administración y Finanzas Habilidades Sensoperceptuales: Visión molar, percepción de la función y utilidades de los puestos.

Motivación: interés por el estudio de las organizaciones y riesgos de inversión.

Técnico científico: Causalidad empírica. Pragmatismo: Detectar factores de riesgo en el puesto y en la inversión.

Motriz: Actividad molar con resistencia a la fatigabilidad.

Cálculo: contable en finanzas y en recursos humanos.

Posibles ocupaciones: Administrador bancario, y financiero, administración de servicios como hoteles.

AREA IV. Comunicación.

Habilidades Sensoperceptuales: Audición y visión de rápida encodificación y decodificación.

Motivación: interés por el estudio de estrategias para informar.

Técnico científico: causalidad empírica del impacto de la información.

Pragmatismo: de función y de utilidad. Motriz: Reflejos rápidos y sostenibles.

Cálculo: de riesgo y peligro.

Posibles ocupaciones: Publicista, director de cine, locución, diseño publicitario, 
dibujante, fotografía, marketing, traductor, producción de TV: Iluminación, camarógrafo, sonido.

AREA V. Laboratorio y Salud.

Habilidades Sensoperceptuales: Significado inductivo mediante la visión, olfato y tacto.

Motivación: interés por preservar la salud física de la comunidad.

Técnico-científico: causalidad empírica. Pragmatismo: de función y de utilidad.

Motriz: motricidad fina.

Cálculo: de proporcionalidad en las mezclas y en las combinaciones.

Posibles ocupaciones: Laboratorista, rehabilitación, obstetra, prótesis dental, visitador médico, técnico farmacéutico, técnico en enfermería, instructor deportivo: aeróbic, fútbol, voley, artes marciales, natación, arbitro, acupuntura, paramédico, primeros auxilios.

AREA VI. Turismo y Hotelería.

Habilidades Sensoperceptuales: Percepción pro y retrospectiva.

Motivación: interés por el cambio, gusto por los viajes y exploraciones.

Técnico científico: causalidad empírica. Pragmatismo: de función y de utilidad.

Motriz: motricidad fina.

Cálculo: de proporcionalidad en las mezclas y en las combinaciones.

Posibles ocupaciones: Laboratorista, rehabilitación, obstetra, prótesis dental, visitador médico, técnico farmacéutico, técnico en enfermería, instructor deportivo: aeróbic, fútbol, vóley, artes marciales, natación, arbitro, acupuntura, paramédico, primeros auxilios.

AREA VII. Mecánica.

Habilidades Sensoperceptuales: Percepción de significados inductivos a partir de la audición.

Motivación: interés por aprender funciones, y manejo de herramientas.

Técnico científico: casualidad empírica de naturaleza física.

Pragmatismo: utilitario.

Motriz: motricidad fina.

Calculo: de perspectiva.

Posibles ocupaciones: Mecánica hidráulica, de explosión, de banco, de reloj, textil, de mantenimiento, de metalurgia, de ajuste, de aviones, soldador, fundidor, modelero, cerrajero, seguridad industrial.

AREA VIII. Construcción y carpintería:

Habilidades Sensopercentuales: Percepción molar a partir de la visión y el tacto.

Motivacion: interés por la simetría y el arte.

Tecnico- científico: casualidad empirica de naturaleza física.

Pragmatismo: logístico, utilitario y funcional.

Motriz: motricidad fina.

Calculo: geométrico y numérico.

Posibles ocupaciones: carpintería, madera y metal, ebanista, albañil. Enchapes, gasfitería, pintor, dibujante técnico, dibujante arquitectónico, topógrafo, decorador, seguridad en construcción civil, jardinería. 




AREA X. Computación e Informática.

Habilidades Sensoperceptuales: Percepción molar a partir de la visión y fineza táctil.

Motivación: interés por resolver problemas relativos a programas y PC.

Técnico-científico: causalidad teórica y empírica.

Pragmatismo: Logístico. Motriz: motricidad fina.

Cálculo: de precisión y anticipación de descargas.

Posibles ocupaciones: Técnico programador, Técnico en PC. Técnico en MAC Mantenimiento de computadoras, Experto en autocad, Webmaster, Técnico en redes.

\begin{abstract}
AREA XI. Artes.
Habilidades Sensoperceptuales: Molaridad Sensorial.

Motivación: interés por expresar conocimiento y emociones a detalle.

Técnico científico: causalidad de impacto.

Pragmatismo: Utilitario. Motriz: motricidad fina.

Cálculo: de precisión y anticipación de descargas.

Posibles ocupaciones: Artes gráficos, Fotomecánica, Serigrafia, Técnico en Offset, Tipografia, Galvano grabador, Artes plásticas: artesanías, joyería, tallado, danzas y bailes, Instructor de teatro, cine canto y música.
\end{abstract}

AREA XII. Técnico en preparación de comidas y bebidas.

Habilidades Sensoperceptuales: Molaridad Sensorial.

Motivación: interés por expresar texturas, gustos, sabores y detalles.

Técnico científico: causalidad de impacto.

Pragmatismo: Logístico y Motriz: motricidad fina.

Cálculo: de cantidades previsión y anticipación.

Posibles ocupaciones: Administrador de restaurantes, gastronomía, pastelería, repostería, Barman, Cheff, industrias alimentarias.

AREA XIII. Belleza y Modas

Habilidades Sensoperceptuales : Molaridad Sensorial, principalmente visión y tacto.

Motivación: interés por expresar y conseguir en otra satisfacción con modelos.

Técnico científico: causalidad de impacto.

Pragmatismo: Logístico y utilitario.

Motriz: motricidad fina.

Cálculo: precisión y anticipación.

Posibles ocupaciones: Cosmética dermatológica, Cosmetólogia, peluquería, estilista, alta costura, lencería, Maquillaje: permanente, manicure, pedicure, confección de vestidos, diseñador de modas, Masajista estética, Modelaje. 


\section{AREA XIV. Producción}

Habilidades Sensoperceptores: Molaridad Sensorial, principalmente visión y olfato.

Motivación: interés por mejorar productos químicos.

Técnico científico: causalidad de impacto.

Pragmatismo: Logístico, funciones y utilitario.

Motriz: motricidad fina.

Cálculo: precisión y anticipación.

Posibles ocupaciones: Química industrial (fabricación de tintes, pinturas, perfumes, jabones, insumos domésticos), Aleaciones (alpaca, oro blanco, platino y otros)

AREA XV. Secretariado.

Habilidades Sensoperceptuales: Molaridad Sensorial.

Motivación: interés por ser útil a las funciones del jefe.

Técnico científico: causal - teórico.

Pragmatismo: Logístico, funciones y utilitario.

Motriz: motricidad fina.

Cálculo: matemático, previsión y anticipación.

Posibles ocupaciones: Secretariado: médico, ejecutivo, legal, administrativo, de banca y finanzas, de comercio, ejecutivo y de administración múltiple.

c) Antes de elaborar los ítemes el constructo, se puso a consideración de un jurado de 20 jueces, integrados por psicólogos orientadores y consejeros vocacionales, con el propósito de verificar si el constructo elaborado era válido para agrupar las carreras técnicas en quince áreas. Se encontró que el cien por cien de los jueces aprobaron el constructo, con recomendaciones de forma y no de contenido los que se ajustaron en el camino.

d) Cada una de las 15 áreas definidas operacionalmente, facilitó encontrar con los trabajadores los indicadores para cada grupo de carreras técnicas. Con los indicadores se elaboró en promedio 50 ítemes por área que hemos denominado escalas.

e) Cada escala se aplicó a trabajadores que como ya dijimos, con no menos de 5 años de experiencia en cada una de las áreas con el propósito de verificar si los ítemes discriminan. Para este grupo se les pidió que señalen en cada uno de los ítemes sí es una característica o un descriptor de su carrera técnica ellos deberían dar sus respuestas en una escala de seis grado que iba desde muy débil o nada hasta el grado seis que indicaba muy fuerte o mucho. Se seleccionaron solo aquellos ítemes que fueron contestados en el grado cinco y seis hasta por el cien por cien de los trabajadores consultados cuyo número fue para algunas carreras como artes y Textilería, 10 técnicos y en otros casos como computación 20 técnicos, en ningún caso se consulto a menos de diez ni a más de 20.

f) Del análisis anterior se detectaron los ítemes que discriminan significativamente desde la óptica de los trabajadores, es necesario recordar que cada ítem se refiere a un rol, función o interés de una carrera técnica. De los 50 ítemes discriminaron 30 como mínimo y 40 como máximo. Los que volvimos a poner a consideración de los jueces ítemes que fueron ratificados al cien por cien.

g) Seleccionado los ítemes se aplicó a un grupo piloto constituido por cien jóvenes entre los 16 a 22 años para efectuar el ajuste semántica. 
h) Finalmente quedó el inventario constituido por 30 ítemes por área, para la aplicación a la muestra de estudio, cada ítem esta constituido por un par de enunciados sobre: roles, funciones o intereses correspondientes a dos áreas de interés, para que el examinado elija uno de ellos, los dos o ninguno. Esta forma de ítem permite que cada indicador se combine con cada uno de las otros incluyendo consigo misma, por ende si un examinado esta definitivamente de acuerdo con una determinada área técnica, habrá efectuado 30 elecciones máximas, la mitad de los itemes corresponden a 1 enunciado identificado como "b", la segunda mítad corresponde a los enuncíados identificados como "a".

i) El Inventario con validez de contenido, obtenida primero cruzando informaciones de personas involucradas en la carrera misma y que identifican al ítem como una característica suya, y por los jueces o expertos que desde su concepción ratificaron cada ítem como parte de cada una de las áreas.

j) De esta forma la prueba quedo expedita para su aplicación a la muestra con tales datos veremos en la siguiente sección los resultados del análisis de ítemes psicométrico, la validez confiabilidad y tipificación.

k) La recolección de datos fue de dos formas, 1) Para el análisis de contenido de los ítemes, la aplicación fue individual a técnicos y personas de oficio que se consideraron exitosos en su trabajo, este juicio partió de los siguientes indicadores, que el trabajador tenga por lo menos 5 años de ejercicio ininterrumpido en un determinado campo técnico u oficio y que manifieste estar satisfecho con su trabajo, a los jueces o psicólogos expertos se les consulto en forma también individual. 2) La aplicación del inventario con validez de contenido fue aplicada a la muestra en grupos no mayores de 25 por vez, cuidando que éstos muestren interés y disposición para el examen, para ello hubo el compromiso de darles sus resultados, lo que se cumplió gracias al apoyo significativo de los colaboradores del estudio.

\section{RESULTADOS}

En esta sección veremos los resultados del análisis de ítemes psicométrico, la validez, la confiabilidad y tipificación.

a) Análisis de ítemes, una vez obtenida la base de datos con los resultados del Inventario, se procedió mediante la correlación tetrácorica a calcular los índices de discriminación de cada uno de los ítemes y para cada área, cuyo resultado se reproduce en el cuadro número 3, donde se podrá leer en la primera columna opción "b" y luego la opción "a". Las siguientes columnas distinguen con un número romano el área técnica, dentro de las cuales debajo del rótulo ítem, se identifica a éstos y debajo de abreviaturas como ELEC, SEGU, identifican el nombre del campo técnico debajo de los cuales se reproduce el índice de discriminación.

Para considerar válido un ítem, éste debe ser igualo mayor de 0.21 , valor que indica que la correlación tetracórico es significativa al 0.05 de margen de error. Hemos encontrado lo siguiente:

En el área de Electricidad y Electrónica (ELEC) solo el ítem I debe ser revisado, no solo por tener correlación baja sino por la dirección negativa.

En el área Seguridad (SEGU) los ítemes 16, 46 y 152, deben ser revisados, no tienen problema de dirección, la dificultad radica en que la discriminación es muy pobre. 
En el área de Administración y Finanzas (AOFI), los ítemes 33, 40 y 138 también deben revisarse.

En el área de Comunicación (COMU) los ítemes 48, 56 y 214 deben revisarse.

En el área de Laboratorio (LABO) los ítemes 65 y 95 deben revisarse.

En el área de Turismo y Hotelería (TUHO) el ítem 81 debe revisarse.

En el área de Mecánica (MECA) los ítemes 91 Y 97 deben revisarse.

En el área de Construcción (CON S) los ítemes 112, 113 deben revisarse.

En el área de Textileria (TEXT) los ítemes 54 y 129 deben revisarse.

En el área de Computación e informática (COIN) el ítem 145 debe revisarse.

En el área de Arte (ARTE) el ítem 131 debe revisarse.

En el área de Comidas y bebidas (COBE) los ítemes 177 y 192 deben revisarse.

En el área de Belleza y modas (BEMO) el ítem 193 debe revisarse.

En el área de Producción (PROD) los ítemes 59, 74, 104, 119 Y 209 deben revisarse.

En el área de Secretaria (SECR) todos los ítemes discriminaron adecuadamente.

b) Validez del Inventario, como viéramos líneas arriba, el inventario fue sometido a un riguroso proceso de construcción, tanto en la agrupación preliminar como en la verificación si estas reflejan a las áreas de carreras tecnológicas, para este propósito consultamos a trabajadores con cinco o más años de experiencia continua, para cada una de las quince áreas y luego de obtener su confirmación pusimos a consideración de diez expertos o jueces, todos ellos psicólogos con experiencia en consejería vocacional, a quienes consultamos primero sobre la tabla de especificaciones, la que fue aprobada por el cien porcino, luego con los indicadores redactamos ítemes, cuyo contenido son roles, funciones e intereses, pertinentes a

cada área tecnológica, ítemes que se aplicaron nuevamente a los técnicos, para seleccionar solamente aquellos que resultaron aprobados por el cien por ciento, estos ítemes también se puso a consideración de los jueces, teniendo como criterio que fuera aprobado por el cien por cien, de esta manera estuvimos seguros que los ítemes exploran los roles, funciones e intereses de cada una de las áreas técnicas.

De esta manera la prueba final contiene 30 ítemes, con enunciados de elección de pares, para cada una de las quince escalas, los que se aplicó a la muestra, con los resultados se procedió a efectuar las correlaciones ínter escalar, con el coeficiente de regresión de Pearson, teniendo como propósito la verificación demostrar que cada escala es independiente, pero a su vez guardan relación significativa entre áreas afines, este análisis fue efectuado con la muestra total, con la muestra de mujeres y con la muestra de varones, encontrando patrones comunes de interpretación, unas nos dicen la proximidad de las áreas y otras no dice que se tratan de campos diferentes por ejemplo en los cuadros 3,4 y 5 se puede leer El área de Electrónica no guarda relación con el área de comunicación, y por lo contrario

Electrónica guarda relación significativa con mecánica, esto indificativa con mecánica, esto indactúan independientemente pero abren la posibilidad de interactuar favorable-mente con otras áreas, es decir que cada escala en la matriz de correlaciones indican que no miden lo mismo demostrando de esta manera que el inventario elaborado tiene validez de constructo. Estas agrupaciones hizo necesario que sometamos el inventario a la prueba varimax de Kaiser, para encontrar los principales componentes rotados que nos sugieran un Inventario con menos áreas que incluyan a otras el resultado puede verse en el cuadro número 6

El componente 1 esta constituido por las áreas: Electrónica-Electricidad, Mecánica, Computación e informática. Que denominaremos Técnico Físicas. 
El Componente 2 está constituido por las áreas: Comunicación, Turismo y hotelería, Seguridad, Administración y finanzas y secretariado. Que denominaremos Técnico organizativas.

El componente 3 esta constituido por las áreas: Producción, Construcción, Textilería, Laboratorio. Que denominaremos Técnicas de producción.

El componente 4 constituido por las regresiones inversas en el componente 1 se agrupa en: Arte, Comida y bebidas y Belleza y modas. Que denominaremos técnicas de servicio.

Obsérvese que ninguna de las escalas ha quedado al margen, siendo las reagrupaciones fáciles de denominar e identificar. Este análisis confirma la validez de constructo del inventario.

c) La confiabilidad del Inventario, para averiguar el grado de error de medición hemos efectuado dos análisis el primero el análisis de consistencia interna utilizando la formula de Kuder Richardson, en Numnally (1973) cuyos resultados presentamos en el cuadro 8, en la que podemos leer que los coeficientes van desde un mínimo de 0.85 hasta un máximo de 0.95 , lo que indica que se trata de 15 escalas con alta a muy alta confiabilidad.

d) Para confirmar estos coeficientes hemos calculado con un grupo de cien examinados la confiabilidad del test retest con un intervalo de tres meses, entre ambas aplicaciones cuyos resultados son análogos a los que encontramos por consistencia interna con fluctuaciones que indican que el inventario evalúa los intereses hacia carreras técnicas con muy bajo error de medición tal como puede verse en el cuadro número 9.

Como se puede observar la Índice fiabilidad (rox) el más bajo va de 0.92 y él más alto llega a la 0.99 razón por la cual podemos afirmar que el Inventario que presentamos presta confiabilidad muy buena con muy bajo error de medición

e) Elaboración de Baremos, ha optado por las normas de puntuaciones $\mathrm{T}$, previamente tuvimos que verificar si en función al sexo de los examinados difieren en los valores promedios de los intereses inventariados para lo cual se calculo las medidas de tendencia central y de variabilidad para describir a los grupos examinados y con la prueba $t$ de diferencia de medias contrasta el resultado, encontrando que de las 15 áreas, en diez de ellas el sexo plantea diferencias, razón por la cual no podemos trabajar con normas de interpretación comunes sino independientes en función al sexo de los examinados.

De las contrataciones efectuadas encontramos:

En el área I: Electricidad-electrónica, los varones presentan un promedio significativamente mayor.

En el área II: Seguridad, los varones presentan un promedio similar al de las mujeres.

En el área III: Administración y finanzas, los varones presentan un promedio similar al de las mujeres significativamente mayor.

En el área IV: Comunicación los varones presentan un promedio similar al de las mujeres.

En el área V: Laboratorio los varones presentan un promedio significativamente menor.

En el área VI: Turismo Hotelería los varones presentan un promedio significativamente menor.

En el área VII: Mecánica los varones presentan un promedio significativamente mayor.

En el área VIII: Construcción los varones presentan un promedio similar al de las mujeres.

En el área IX: Textileria los varones presentan un promedio similar al de las mujeres.

En el área X: Computación-informática los varones presentan un promedio significativamente mayor. 
En el área XI: Arte los varones presentan un promedio significativamente menor.

En el área XII: Comidas y bebidas los varones presentan un promedio significativamente menor.

En el área XIII: Belleza y modas los varones presentan un promedio significativamente menor.

En el área XIV: Producción los varones presentan un promedio significativamente mayor. En el área XV: Secretariado los varones presentan un promedio significativamente menor.

Los resultados descritos se pueden ver en el cuadro número 10. 
Tabla Nro. 01 de distribución poblacional en función a la edad, sexo y agrupados por conos

\begin{tabular}{|c|c|c|c|c|c|c|c|c|c|c|c|c|c|}
\hline & \multicolumn{2}{|c|}{ Centro } & \multicolumn{2}{|c|}{ Norte } & \multicolumn{2}{|c|}{ Sur } & \multicolumn{2}{|c|}{ Estc } & \multicolumn{2}{|l|}{ Oes } & \multicolumn{2}{|c|}{ Sudo-total } & \multirow[t]{2}{*}{ Total } \\
\hline & Varón & Mujer & Varón & Mujc & Varón & Mujer & arón & Mujer & arón & jer & Varón & $\mathrm{Muj}$ & \\
\hline & & & 70048 & & $95\{20$ & 0 & 77137 & 82 & 1160 & 385 & B1509 & & \\
\hline & 760 & & & & & & & & & & & & \\
\hline & 645 & 108450 & 157698 & 167535 & 187854 & 0 & 150690 & 159 & 227. & Trove & 6221 & 666751 & 1289 \\
\hline
\end{tabular}

Cuadro Nro. 02 Centros y tipos de Capacitación técnica

\begin{tabular}{|c|c|c|c|c|c|}
\hline & $\begin{array}{c}\text { Centro de Educación } \\
\text { Ocupacional } \\
\text { C.E.O. }\end{array}$ & $\begin{array}{l}\text { Instituto } \\
\text { Superior } \\
\text { Pedagógico }\end{array}$ & $\begin{array}{l}\text { Instituto } \\
\text { Superior } \\
\text { Tecnológieo }\end{array}$ & Escuelas & $\begin{array}{l}\text { Escuela } \\
\text { Pre } \\
\text { Militar }\end{array}$ \\
\hline Discrio Alta Costura & 1 & 1 & & 3 & \\
\hline Mecánica & I & & 7 & & \\
\hline Computación & 4 & 30 & 40 & & 1 \\
\hline Secretariado & 5 & 2 & 13 & & \\
\hline Gastronomia & & 3 & & 5 & \\
\hline Hoteleria - Turismo & & 3 & & 5 & \\
\hline Idiomas & 2 & 24 & & 4 & \\
\hline Educaciỏn & 3 & 14 & & & \\
\hline Cosmetologia & & & 1 & & \\
\hline $\begin{array}{l}\text { Contabilidad } \\
\text { Administración }\end{array}$ & & 12 & & 2 & \\
\hline Enfermeria & & 1 & & & \\
\hline Farmacia & & 1 & & & \\
\hline Diseño Gráfico & & 7 & & 3 & \\
\hline Marketing & & & & & \\
\hline TOTAL & 16 & 39 & 43 & 10 & 1 \\
\hline
\end{tabular}


TABLA N ${ }^{\circ}$ 3: VALIDEZ DE ITEMS POR EL INDICE DE DISCRIMINACION

\begin{tabular}{|c|c|c|c|c|c|c|c|c|c|c|c|c|c|c|c|c|}
\hline & \multicolumn{2}{|l|}{ II } & \multicolumn{2}{|l|}{ IIII } & \multicolumn{2}{|l|}{ IV } & \multicolumn{2}{|l|}{ V } & \multicolumn{2}{|l|}{ VI } & \multicolumn{2}{|l|}{ VII } & \multicolumn{2}{|l|}{ VIIII } \\
\hline & IFII & FIEC & ITEMII & SEGI & ITEM & ADFI & IIEM & cosit & ITEN & LABO & ITEM & ТЕНо & ITEM & MECA & ITEN & $C 0$ \\
\hline & $T$ & 0.2 & 16 & 0.49 & 31 & 0,37 & 40. & 075 & 61 & 0.38 & 76 & 0,33 & 91 & 0.11 & 106 & \\
\hline & 1 & 0,41 & 79 & & 32 & & +7 & & & 0.32 & & 0.25 & & & 07 & 36 \\
\hline & 3 & 0.46 & & & & & 48 & & & & 78 & & 93 & & 08 & 41 \\
\hline & 4 & 0 & - & & 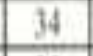 & & & & & & & & & & 109 & 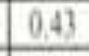 \\
\hline & 5 & & 7 & & 35 & & 9 & & & & 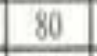 & & & & 110 & 39 \\
\hline & 6 & & 21 & & & & 7 & & & & & & & & III & \\
\hline & 7 & & $=$ & & 37 & & $=$ & & & & & & & & 2 & 47 \\
\hline & 8 & & 23 & & & & & & & & & & & & 13 & 0.12 \\
\hline & - & & 3 & & & & & & & & & & 2 & & - & 0.4 \\
\hline & 10 & 0 & 25 & & $=$ & & 3 & & & & & & & & 13 & 41 \\
\hline & II & & & & 4 & & 3 & & & & & & 101 & & 6 & 0.2 \\
\hline & 12 & 0.5 & 27 & 0 & & & & & & & & 0.47 & 102 & 0.86 & III & 37 \\
\hline & 13 & 0.8 & 28 & 0 & 4 & 10 & 5 & & 7 & & & $\pi$ & 103 & & 118 & 0.46 \\
\hline & 14 & & & & & & 5 & & & & & & 104 & & 119 & 0.37 \\
\hline & 15 & 0. & 3 & & 4 & 0.1 & 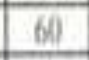 & & & & & & 105 & & 12 & 47) \\
\hline & 1 & 0. & 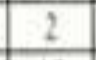 & & 3 & 0. & 4 & & & & & & 4 & & & 0.42 \\
\hline & 16 & 0 & $\sqrt{17}$ & & & 0.4 & - & & & & $=$ & & 2 & & 23 & 4) \\
\hline & 3 & 0. & 32 & & 3 & 0.2 & 1 & & & & & & & & 8 & 45 \\
\hline & 46 & 0.1 & 47 & & & 0. & 4 & & & & - & - & 52 & & 3 & 0.46 \\
\hline & $6 !$ & $\overline{0.3}$ & 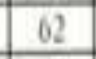 & & & 03 & & & & & 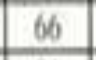 & & - & & 8 & 26 \\
\hline & 76 & 0. & 7 & 0 & 77 & 0.59 & 78 & & 80 & & 81 & 70 & 82 & 13 & 33 & 0.47 \\
\hline & $9 !$ & 0.5 & 92 & & & 0.3 & $\ldots$ & & & & 96 & & 97 & .0 & 08 & 0.2 \\
\hline & 100 & 0.5 & 107 & 0.8 & & 0,73 & 109 & & 110 & 0. & III & & 112 & $\pi$ & 1 & 2.07 \\
\hline & 121 & 0.6 & 122 & 0. & 123 & \begin{tabular}{|l|}
0.39 \\
\end{tabular} & 124 & & 125 & 0 & 126 & 0.54 & 127 & 22 & 28 & 0.38 \\
\hline & 119 & 0. & 137 & & & 0.22 & 139 & & 140 & 0 & 141 & 0.56 & 142 & 67 & 143 & 0.55 \\
\hline & 151 & 0.72 & 152 & 0.1 & 153 & 0.58 & 154 & & 155 & 0. & 156 & 0,03 & 131 & 0.86 & 158 & 0,32 \\
\hline & 166 & 0.58 & 167 & & 168 & 0.3 & 169 & 0 & 170 & 0. & 171 & 0.48 & 172 & 0.8 & 173 & 0.29 \\
\hline & 181 & 0.84 & 182 & & 183 & 0.42 & 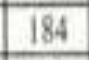 & & 103 & Q. & 186 & & 187 & 59 & 188 & 0.41 \\
\hline & 190 & 0.6 & 197 & 0.38 & 19: & 0.52 & 199 & 0,4 & 200 & 0.32 & 201 & & 202 & 30 & 203 & 0.23 \\
\hline & $=$ &  & 21 & & $\sqrt{4}$ & 0.38 & 21 & 0.14 & 215 & 0.53 & 216 & 0.51 & 217 & 0.48 & 218 & 0.46 \\
\hline
\end{tabular}




\begin{tabular}{|c|c|c|c|c|c|c|c|c|c|c|c|c|c|c|}
\hline \multicolumn{3}{|c|}{$1 \mathrm{Ix}$} & \multicolumn{2}{|c|}{$\mathrm{x}$} & \multicolumn{2}{|c|}{ XI } & \multicolumn{2}{|c|}{ XII } & \multicolumn{2}{|l|}{ XIII } & \multicolumn{2}{|l|}{ XIV } & \\
\hline & ITEMI & TEXT & ITEM & COIv & EMI & ARTE & ITEMI & COBE & ITEM & BEMO & ITEM & 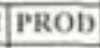 & & Is \\
\hline & 121 & 80 & 73 & 0.55 & 151 & & 166 & 072 & 181 & 08 & & 0.97 & & \\
\hline & 122 & & & & & & & & & & & & & \\
\hline & 123 & & & & & & & & & & & & & \\
\hline & 124 & & & & & & & & & & & & & \\
\hline & 125 & 0 & & & & & & & & & & & & \\
\hline & 126 & & 1. & & & & & & & & & & & \\
\hline & 127 & & & & 1 & & & & & & & & & \\
\hline & 12 & & & & & & & & & & & & & \\
\hline & 12 & & & & & & & & & & & & & \\
\hline & 130 & & & & & & & & & & & & & \\
\hline & 131 & 0. & & & If & & & & & & & & & \\
\hline & 132 & 0 & 14 & 0.8 & 16. & & I & & & & & & & \\
\hline & 13 & & & 0 & II & & & & & & & & & \\
\hline & 13. & & & & & & & & & & & & & \\
\hline & 135 & 0.4 & 150 & 0. & 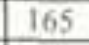 & & & & & & & & & \\
\hline & 9 & 0.5 & 11 & 04 & 11 & & & & & & & & & \\
\hline & 24 & & & 0.4 & 2 & & & & & & & & & \\
\hline & 39 & & & & & & & & & & & & & \\
\hline & 54 & 0 & & & & & & & & & & & & \\
\hline & 69 & 0.2 & 7 & & & & & & & & & & & \\
\hline & 84 & 03 & & 0 & & & & & & & & & & \\
\hline & 99 & 0.4 & II & 0. & on & & & & & & & & & {$[0$} \\
\hline & 114 & 0.2 & & & & & & & & & & & & \\
\hline & 129 & 0.1 & & & & & & & & & 14 & 18 & 35 & 1284 \\
\hline & 144 & & & & & & & & & & & & 0 & \\
\hline & 159 & 0.43 & 10 & 0.13 & & & & & & & & & 55 & 0.2 \\
\hline & 174 & 0.35 & 1 & 0.8 & 176 & & & & & & & & & \\
\hline & 189 & D. & & 0.72 & 191 & & 1 & 0.14 & 193 & & 24 & & 5 & \\
\hline & 20 & 0. & & & 2 & & & & 21 & +. & 09 & 31 & 0 & 0.4 \\
\hline & 219 & 0.51 & 220 & 0.61 & 22 & Nis & 22 & 0.4 & 22 & 0.2 & 22 & 0.62 & 225 & 0.2 \\
\hline
\end{tabular}

Cuadro Nro. 04 Matriz de correlación inter escalar del total de la muestra examinada

\begin{tabular}{|c|c|c|c|c|c|c|c|c|c|c|c|c|c|}
\hline ELEC $_{\text {. }}$ & 1 & & & & & & & & & & & & \\
\hline SEGU & 0.06 & 1 & & & & & & & & & & & \\
\hline ADFI & 0.13 & 0.15 & 1 & & & & & & & & & & \\
\hline СомU & -0.05 & 0.35 & 0.26 & 1 & & & & & & & & & \\
\hline L.ABO & -0.08 & 0.27 & .0 .01 & -0.05 & I & & & & & & & & \\
\hline TUНО & -0.17 & 0.39 & 0.19 & 0.30 & 0.28 & 1 & & & & & & & \\
\hline MECA & 0.75 & 0.03 & 0.03 & -0.13 & -0.05 & -0.20 & 1 & & & & & & \\
\hline CONS & 0.24 & 0.19 & 0.02 & 0.04 & 0.06 & 0.07 & 0.35 & 1 & & & & & \\
\hline TEXT & -0.13 & -0.05 & $-0,05$ & -0.01 & 0.11 & -0.02 & 0.00 & 0.24 & & & & & \\
\hline COIN & 0.48 & 0.05 & 0.23 & 0.03 & -0.05 & 0,01 & 0.31 & $0.03-0.15$ & 1 & & & & \\
\hline ARTE & -0.35 & 0.33 & -0.01 & 0.35 & 0.14 & 0.39 & -0.32 & 0.170 .24 & -0.24 & 1 & & & \\
\hline COBE & -0.25 & 0.10 & 0.01 & 0.07 & 0.25 & 0.22 & -0.17 & $0.12 \quad 0.32$ & -0.27 & 0.31 & 1 & & \\
\hline BEMO & -0.49 & 0.09 & -0.10 & 0.09 & 0.18 & 0.25 & -0.44 & $-0.02 \quad 0.27$ & $-0,35$ & 0.51 & 0.40 & 1 & \\
\hline PROD & 0.37 & 0.14 & 0.04 & -0.06 & 0.36 & 0.01 & 0.41 & 0.340 .16 & 0.18 & -0.02 & 0.10 & -0.08 & 1 \\
\hline SECR & -0.13 & 0.07 & 0.32 & 0.21 & 0.05 & 0.16 & -0.17 & 0.030 .13 & 0.00 & 0.16 & 0.21 & 0.19 & 0.02 \\
\hline
\end{tabular}


Cuadro Nro. 05 Matriz de correlación inter escalar obtenida de 148 varones de Lima

\begin{tabular}{|c|c|c|c|c|c|c|c|c|c|c|c|c|c|c|c|}
\hline AREAS & EEC & SEGU & ADEI & cove & LABO & Tuto: & MECA & covs & TEX & coin & ABTE & COBE & AEMOI & PUODSE, & $C D$ \\
\hline ELEC & 1 & & & & & & & & & & & & & & \\
\hline SEOU & 0.09 & 1 & & & & & & & & & & & & & \\
\hline ADF1 & 0.23 & 0.33 & 1 & & & & & & & & & & & & \\
\hline Comu & 0.11 & 0.39 & 0.37 & 1 & & & & & & & & & & & \\
\hline LABO & 0.06 & 0.34 & 0.16 & 0.11 & 1 & & & & & & & & & & \\
\hline TИно & 0.06 & 0.45 & 0.34 & 0.41 & 0.36 & 1 & & & & & & & & & \\
\hline MECA & 0.66 & 0.14 & 0.16 & 0.05 & 0.08 & 0.06 & I & & & & & & & & \\
\hline cons & 0.42 & 0.35 & 0.27 & 0.20 & 0.21 & 0.23 & 0.50 & 1 & & & & & & & \\
\hline TEXT & -0.05 & 0.15 & 0.08 & 0.04 & 0.35 & 0.14 & 0.11 & 0.28 & 1 & & & & & & \\
\hline COIN & 0.43 & 0.14 & 0.28 & 0.24 & 0.00 & 0.23 & 0.27 & 0.15 & -0.07 & 1 & & & & & \\
\hline ARTE & -0.07 & 0.40 & 0.25 & 0.39 & 0.29 & 0,45 & -0.02 & 0.30 & 0.34 & -0.05 & 1 & & & & \\
\hline СОВЕ & -0.07 & 0.21 & 0.14 & 0.13 & 0.44 & 0.27 & -0.02 & 0.13 & 0.36 & -0.07 & 034 & 1 & & & \\
\hline BEMO & -0.16 & 0.11 & 0.06 & -0.03 & 0.25 & 0.10 & -0.13 & 0.09 & 0.39 & -0.25 & 0.42 & 0.36 & 1 & & \\
\hline PROD & 0.30 & 0.23 & 0.27 & 0.23 & 0.45 & 0.26 & 0.42 & 0.38 & 0.40 & 0.13 & 0.25 & 0.30 & 0.20 & 1 & \\
\hline SECR & -0.06 & 0.14 & 0.17 & 0.22 & 0.22 & 022 & -0.01 & 0.15 & 0.36 & 0.02 & 0.27 & 0.32 & 0.31 & 0.27 & 1 \\
\hline
\end{tabular}

Cuadro Nro. 06 Matriz de correlación inter escalar obtenida de 288 mujeres de Lima



Para encontrar en cuantos componentes podenos organizar una versión simplificada del inventario hemos utilizado Método de extraeción: Analisis de componentes principales. Método de rotación: Normalización Varimax con Kaiser encontrando tres componentes integrados las componentes I seis direas, el componente 2 por cinco àreas y el componente tres por cuatro áreas, indicando que a la rotación han convergido 5 iteraciones 


\section{Cuadro Nro. 7 De Matriz de componentes rotados}

\begin{tabular}{lccc} 
& \multicolumn{3}{c}{ Componente } \\
& 1 & 2 & 3 \\
\hline AREA 1 & 0.846 & & \\
AREA 7 & 0.756 & & \\
AREA 13 & -741 & & \\
AREA 10 & 0.659 & & \\
AREA 11 & -501 & & \\
AREA 12 & -486 & & \\
AREA 4 & & 0.704 & \\
AREA 6 & & 0.666 & \\
AREA 2 & & 0.639 & \\
AREA 3 & & 0.599 & \\
AREA 15 & & 0.452 & \\
AREA 14 & & & 0.715 \\
AREA 8 & & & 0.655 \\
AREA 9 & & & 0.540 \\
AREA 5 & & & \\
\hline
\end{tabular}

Tabla $\mathrm{N}^{*} 8$

De confiabilidad por el método de la Consistencia interna del Inventario para carreras técnicas (IINCATEC 2001)

\begin{tabular}{cll}
\hline Area & \multicolumn{1}{c}{ Nombre } & r11 \\
\hline 1 & Elec. & 0.95 \\
2 & Seguridad & 0.85 \\
3 & Adminis/Finan & 0.87 \\
4 & Comunicación & 0.85 \\
5 & Labotatorio & 0.90 \\
6 & TurismiHoteler & 0.88 \\
7 & Mecinica & 0.95 \\
8 & Construcción & 0.83 \\
9 & Textileria & 0.85 \\
10 & Computation /nformática & 0.94 \\
11 & Arte & 0.92 \\
12 & Técnico en Comida /Bebidas & 0.92 \\
13 & Belleza $y$ Moda & 0.95 \\
14 & Producción & 0.88 \\
15 & Secretariado & 0.89 \\
\hline
\end{tabular}


Tabla N*9

De confiabilidad por el método del test retest Inventario para earreras técuicas (IINCATEC 2001)

\begin{tabular}{clcc} 
Area & Nombre & rll & Rox \\
\hline 1 & Elec. & 0.98 & 0.99 \\
2 & Seguridad & 0.89 & 0.94 \\
3 & Adminis/Finan & 0.90 & 0.95 \\
4 & Comunicación & 0.88 & 0.94 \\
5 & Laboratorio & 0.92 & 0.96 \\
6 & Turismo /Hoteleria & 0.90 & 0.95 \\
7 & Mecánica & 0.98 & 0.99 \\
8 & Construcción & 0.89 & 0.94 \\
9 & Textileria & 0.90 & 0.95 \\
10 & Cormputation /Informática & 0.96 & 0.98 \\
11 & Arte & 0.96 & 0.98 \\
12 & Técnico en Comidas/Bebidas & 0.98 & 0.99 \\
13 & Belleza y Moda & 0.96 & 0.98 \\
14 & Producción & 0.91 & 0.95 \\
15 & Secretariado & 0.92 & 0.92 \\
\hline
\end{tabular}


CUADRO Nro. 10

MEDIDAS DE TENDENCIA CENTRAL Y RAZON CRITICA DE DIFERENCIAS DE MEDIAS POR ÁREAS EN FUNCION AL GENERO IINCATEC-2001

\begin{tabular}{|c|c|c|c|c|c|c|c|c|c|c|c|c|c|c|}
\hline \multirow{2}{*}{  } & \multicolumn{2}{|c|}{ ELEC } & \multicolumn{2}{|c|}{ SEGU } & ADFI & \multicolumn{2}{|c|}{ COMU } & \multicolumn{2}{|c|}{ LABO } & \multicolumn{2}{|c|}{ ТИНО } & \multicolumn{3}{|c|}{ MECA } \\
\hline & V & M & V & M & V & $M$ & V & M & V & $M$ & V & M & V & M \\
\hline Media & 18.02 & $\pi 1.96$ & 17.60 & 17.93 & 17.55 & 17.34 & 17.21 & 17,48 & 13.21 & 15.20 & 18.25 & 20.86 & 18.05 & 11.17 \\
\hline Emor típico & 0.35 & 0.27 & 0.32 & 0.23 & 0.32 & 0.27 & 0.50 & 0.26 & 0.35 & 0.30 & 0.35 & 0.24 & 0.44 & 0.30 \\
\hline Mediana & 18 & 11 & 18 & 18 & 17 & 17 & 17 & 17 & 13 & 15 & 18 & 21 & 18 & 11 \\
\hline Moda & 19 & 9 & 17 & 17 & 16 & 16 & 16 & 17 & 12 & 13 & 14 & 21 & 15 & 8 \\
\hline Desviación estindar & 4.73 & 4.50 & 4.27 & 3.98 & 4.27 & 4.62 & 4,10 & 4.39 & 4.60 & 5.06 & \begin{tabular}{|l|}
4.69 \\
\end{tabular} & 4.14 & 5.93 & 5.09 \\
\hline $\begin{array}{c}\text { Varianta de la } \\
\text { muestra }\end{array}$ & 22.40 & 20.23 & 18.27 & 15.85 & 18.26 & 21.3 & 16.78 & 19.24 & 21.95 & 25.61 & 22,00 & 17.15 & 35.16 & 25.91 \\
\hline Curtosis & -0.22 & 0.20 & 0.51 & -0.20 & 0.63 & -0.59 & 0.40 & -0.38 & 0.82 & $.0,46$ & 0.08 & -0.28 & -0.64 & 0.28 \\
\hline $\begin{array}{c}\text { Coeficiente de } \\
\text { asimetria }\end{array}$ & -0.18 & 0.63 & 0.01 & 0.22 & 0.36 & 0.05 & 0.16 & 0.10 & 0.60 & 0.36 & \begin{tabular}{|l|}
0,13 \\
\end{tabular} & -0.25 & -0.03 & 0.71 \\
\hline Rungo & 24 & 25 & 27 & 22 & 25 & 23 & 24 & 23 & 26 & 25 & 26 & 20 & 27 & 27 \\
\hline Minimo & 5 & 2 & 3 & 8 & 5 & 6 & 6 & 7 & 3 & 3 & 4 & 10 & 3 & 2 \\
\hline Máximo & 29 & 27 & 30 & 30 & 30 & 29 & 30 & 30 & 29 & 28 & 30 & 30 & 30 & 29 \\
\hline Suma & 3315 & 3444 & 3238 & 5165 & 3229 & 4995 & 3167 & 5035 & 2431 & 4378 & 3358 & 6008 & 3321 & 3218 \\
\hline Cuenta & 184 & 288 & 184 & 288 & 184 & 288 & 184 & 288 & 184 & 288 & 184 & 288 & 184 & 288 \\
\hline Diferencia & 6.06 & & -0.34 & & 0.21 & & -0.27 & & -1.99 & & -2.61 & & 6.88 & \\
\hline Ertor Standand & 0.44 & & 0.39 & & 0.42 & & 0.40 & & 0.46 & & 0.42 & & 0.53 & \\
\hline 1 & 13.83 & & -0.86 & & 0.49 & & .0 .68 & & -4.36 & & -6.17 & & 12.97 & \\
\hline Decisión & Hi & & $\mathrm{Ho}_{0}$ & & ho & & ho & & hi & & hi & & hi & \\
\hline
\end{tabular}




\begin{tabular}{|c|c|c|c|c|c|c|c|c|c|c|c|c|c|c|c|c|}
\hline \multirow{2}{*}{$\begin{array}{l}\text { AREA } \\
\text { SEXO }\end{array}$} & \multicolumn{2}{|c|}{ CONS } & \multicolumn{2}{|c|}{ TEXT } & \multicolumn{2}{|c|}{ COIN } & \multicolumn{2}{|c|}{ ARTE } & \multicolumn{2}{|c|}{ COBE } & \multicolumn{2}{|c|}{ BEMO } & \multicolumn{2}{|c|}{ PROD } & \multicolumn{2}{|c|}{ SECR } \\
\hline & V & $\bar{M}$ & v & $\bar{M}$ & v & $\bar{M}$ & $\bar{v}$ & $\bar{M}$ & $\mathrm{v}$ & $\mathrm{M}$ & $\bar{v}$ & $\bar{M}$ & $\bar{v}$ & $\mathrm{M}$ & $\bar{v}$ & $\overline{\mathrm{M}}$ \\
\hline Media & 17.26 & 16.66 & 11.92 & 12.43 & 20.38 & 18.71 & 15.90 & 19.62 & 14.58 & 15.7 & 11.7 & 17.51 & 14.78 & 13.2 & 13.79 & 14.76 \\
\hline Error típico & 0.32 & 0.22 & 0.33 & 0.25 & 0.40 & 0.36 & 0.37 & 0.30 & 0.39 & 0.32 & 0.4 & 0.34 & 0.34 & 0.26 & 0.34 & 0.29 \\
\hline Mediana & 17.5 & 16 & 12 & 12 & 21 & 19 & 15 & 20 & 14 & 15 & 11 & 18 & 15 & 13 & 14 & 15 \\
\hline Moda & 20 & 15 & 14 & 12 & 17 & 18 & 13 & 20 & 14 & 13 & 12 & 21 & 15 & 13 & 14 & 16 \\
\hline $\begin{array}{l}\text { Desviación } \\
\text { estandar }\end{array}$ & 4.38 & 3.75 & 4,42 & 4.22 & 5.44 & 6.10 & 4.98 & 5.04 & 5.31 & 5.43 & \begin{tabular}{|l|l}
5.42 \\
\end{tabular} & 5.84 & 4.66 & 4.33 & 4.64 & 4.84 \\
\hline $\begin{array}{l}\text { Varianza de la } \\
\text { muestra }\end{array}$ & 19.20 & 14.04 & 19.54 & 17,79 & 29.55 & 37.21 & 24.83 & 25.44 & 28.21 & 29.5 & \begin{tabular}{|l|l|}
29.4 &
\end{tabular} & 34,06 & 21.70 & 18.8 & 21.54 & 23.42 \\
\hline Curtosis & 0.24 & -0.36 & $\mid 0.87$ & \begin{tabular}{|l|}
0.03 \\
\end{tabular} & $-0,30 \mid$ & -0.85 & 0.33 & -0.54 & 0.37 & -0.2 & \begin{tabular}{|l|l}
-0.2 & \\
\end{tabular} & -0.51 & 0.83 & 0.43 & 0.42 & 0.07 \\
\hline $\begin{array}{c}\text { Coeficiente de } \\
\text { asimetria }\end{array}$ & 0.11 & 0.12 & 0.53 & 0.40 & -0.24 & -0.07 & 0.36 & -0.16 & 0.37 & 0.36 & 0.39 . & -0.25 & $-0,06$ & 0.44 & $-0,06$ & 0.21 \\
\hline Rango & 25 & 19 & 24 & 23 & 24 & 27 & 29 & 24 & 29 & 27 & 28 & 28 & 28 & 25 & 28 & 26 \\
\hline Minimo & 4 & 8 & 2 & 3 & 6 & 3 & 1 & 6 & 1 & 3 & 0 & 2 & 1 & 3 & 1 & 3 \\
\hline Máximo & 29 & 27 & 26 & 26 & 30 & 30 & 30 & 30 & 30 & 30 & 28 & 30 & 29 & 28 & 29 & 29 \\
\hline Suma & 3175 & 4797 & 2194 & 3581 & 3750 & 5388 & 2925 & 5650 & 2683 & 4513 & 2153 & 5043 & 2720 & 3799 & 2538 & 4251 \\
\hline Cuenta & 184 & 288 & 184 & 288 & 184 & 288 & 184 & 288 & 184 & 288 & 184 & 288 & 184 & 288 & 184 & 288 \\
\hline Diferencia & 0.60 & & -0.51 & & 1.67 & & -3.72 & & -1.09 & & 5.81 & & 1.59 & & -0.97 & \\
\hline Error Stundand & 0.39 & & 0.41 & & 0.54 & & 0.47 & & 0.51 & & 0.53 & & 0.43 & & 0.45 & \\
\hline $\mathrm{T}$ & 1.53 & & -1.24 & & 3.11 & & -7.88 & & -2.15 & & $-11,02$ & & 3.72 & & -2.17 & \\
\hline Decisión & ho & & ho & & hi & & hi & & hi & & hi & & hi & & hi & \\
\hline
\end{tabular}


A continuación presentamos los baremos en puntajes para cada una de las escalas en función al género

Tabla Nro. 11 de conversión de puntajes directos a puntajes T obtenido con una muestra de 184 varones de Lima metropolitana

\begin{tabular}{|c|c|c|c|c|c|c|c|}
\hline \multirow[b]{2}{*}{ Areas } & \multicolumn{7}{|c|}{ Puntaje T } \\
\hline & 20 a 29 & $30=39$ & 40 a 44 & 45 a 54 & 55 a 59 & 60 a 69 & 70 a 80 \\
\hline ELEC. & 0 a 8 & 9 a 13 & 14 a 15 & $16=20$ & 21 a 22 & $23 a 27$ & 28 a 30 \\
\hline SEGU & 0 a 8 & 9 a 13 & $14 a 15$ & $16 \times 19$ & $20=21$ & 22 a 25 & 26 a 30 \\
\hline ADFI & $0 \square 9$ & 10 a 13 & 14 a 15 & $16 \square 19$ & $20+21$ & 22 a. 26 & 27 a 30 \\
\hline COMU & $0=9$ & 10 a 13 & 14 a 15 & 16 a 19 & 20 a 21 & 22 a 25 & 26 a 30 \\
\hline LABO & 0 a 3 & $4 a 8$ & $9=10$ & 11 a 15 & 16 a 17. & 18 a 22 & 23 a 30 \\
\hline туно & $0 a 8$ & 9 a 13 & 14 a 15 & $16 a 20$ & $21 \times 22$ & 23 a 27 & $28=30$ \\
\hline MECA & 0 as & $6 a 11$ & $12 \times 14$ & 15 a 20 & 21 a 23 & 24 a 28 & 29 a 30 \\
\hline CONS & 0 a 8 & $9 a 12$ & 13 a 14 & 15 a 19 & $20 \times 21$ & 22 a 25 & 26 a 30 \\
\hline TEXT & 0 a 2 & 3 a 7 & 8 a 9 & 10 a 13 & 14 a 16 & 17 a 20 & 21 a 30 \\
\hline COIN & 0 a 9 & 10 a 14 & $15 \times 17$ & $18 a 22$ & $23 a 25$ & 26 a 28 & 29 a 30 \\
\hline ARTE & 0 as & 6 a 10 & 11 a 13 & 14 a 18 & 19 a 20 & 21 a 25 & 26 a 30 \\
\hline COBE & $0 \mathrm{a} 3$ & 4 a 9 & $10 \mathrm{a} 11$ & $12 \times 16$ & 17 a 19 & $20 a 24$ & 25 a 30 \\
\hline BEMO & 0 a 1 & 2 a 6 & $7: 8$ & 9 a 14 & 15 a 17 & 18 a 22 & 23 a 30 \\
\hline PROD & ous & 6 a 9 & 10 a 12 & 13 a 16 & 17 a 19 & 20 a 23 & 24 a 30 \\
\hline SECR & 0.4 & 5 a 8 & 9 a 12 & 13 a 15 & 16 a 18 & 19 a 22 & 23 a 30 \\
\hline Puntaje $\mathrm{T}$ & $20+29$ & 30 a 39 & 40 a 44 & 45 a 54 & 55 a 59 & 60 a 69 & 70 a 80 \\
\hline Categoria & $\begin{array}{c}\text { muy poco } \\
\text { interés }\end{array}$ & $\begin{array}{l}\text { poco } \\
\text { interes }\end{array}$ & $\begin{array}{l}\text { tendencia } \\
\text { al desinierés }\end{array}$ & $\begin{array}{c}\text { Interés } \\
\text { Ambivalencia }\end{array}$ & $\begin{array}{l}\text { tendencia } \\
\text { al interés }\end{array}$ & $\begin{array}{l}\text { alto } \\
\text { interés }\end{array}$ & $\begin{array}{l}\text { muy alto } \\
\text { interes }\end{array}$ \\
\hline
\end{tabular}


Tabia Nro. 11 de conversión de puntajes directos a puntajes T obtenido con una muestra de 288 mujeres de Lima metropolitana

\begin{tabular}{|c|c|c|c|c|c|c|c|}
\hline \multirow[b]{2}{*}{ Areas } & \multicolumn{7}{|c|}{$P$ u $n t a j$ e $T$} \\
\hline & 20 a 29 & 30 a 39 & 40 a 44 & 45 a 54 & 55 a 59 & 60 a 69 & 70 a 80 \\
\hline ELEC. & 0 a 2 & 3 a 7 & 8 a 9 & 10 a 13 & 14 a 16 & 17 a 20 & 21 a 30 \\
\hline SEGU & 0 a 9 & 10 a 13 & 14 a 15 & 16 a 19 & 20 a 21 & 22 a 25 & 26 a 30 \\
\hline ADFI & 0 a 8 & 9 a 12 & 13 a 15 & 16 a 19 & 20 a 21 & 22 a 26 & 27 a 30 \\
\hline $\mathrm{COMU}$ & 0 a 8 & 9 a 13 & 14 a 15 & 16 a 19 & 20 a 21 & 22 a 26 & 27 a 30 \\
\hline LABO & 0 a 5 & 6 a 10 & 11 a 12 & 13 a 17 & 18 a 20 & 21 a 25 & 26 a 30 \\
\hline TUHO & 0 a 12 & 13 a 16 & 17 a 18 & 19 a 22 & 23 a 24 & 25 a 28 & 29 a 30 \\
\hline MECA & 0 a 1 & 2 a. 5 & 6 a 8 & 9 a 13 & 14 a 16 & 17 a 21 & 22 a 30 \\
\hline CONS & 0 a 8 & 9 a 12 & 13 a 14 & 15 a 18 & 19 a 20 & 21 a 23 & 24 a 30 \\
\hline TEXT & 0 a 3 & 4 a 8 & 9 a 10 & 11 a 14 & 15 a 16 & 17 a 20 & 21 a 30 \\
\hline COIN & 0 a 6 & 7 a 12 & 13 a 15 & 16 a 21 & 22 a 24 & 25 a 28 & 29 a 30 \\
\hline ARTE & 0 a 9 & 10 a 14 & 15 a 16 & 17 a 21 & 22 a 24 & 25 a 28 & 29 a 30 \\
\hline COBE & 0 a 4 & 5 a 10 & 11 a 12 & $13 \times 18$ & 19 a 21 & 22 a 26 & 27 a 30 \\
\hline BEMO & 0 a 5 & 6 a 11 & 12 a 14 & 15 a 20 & 21 a 23 & 24 a 28 & 29 a 30 \\
\hline PROD & 0 a 3 & 4 a 8 & 9 a 10 & 11 a 15 & 16 a 17 & 18 a 22 & 23 a 30 \\
\hline SECR & 0 a. 4 & 5 a 9 & 10 a 12 & 13 a 16 & 17 a 19 & 20 a 24 & 25 a 30 \\
\hline Puntaje $T$ & 20 a 29 & 30 a 39 & 40 a 44 & 45 a 54 & 55 a 59 & 60 a 69 & 70 a 80 \\
\hline Categoría & $\begin{array}{c}\text { muy poco } \\
\text { interés }\end{array}$ & $\begin{array}{c}\text { poco } \\
\text { interés }\end{array}$ & $\begin{array}{l}\text { tendencia } \\
\text { al desinterès }\end{array}$ & $\begin{array}{c}\text { Interés } \\
\text { Ambivalencia }\end{array}$ & $\begin{array}{l}\text { tendencia } \\
\text { al interés }\end{array}$ & $\begin{array}{c}\text { alto } \\
\text { interés }\end{array}$ & $\begin{array}{c}\text { muy alto } \\
\text { interés }\end{array}$ \\
\hline
\end{tabular}




\section{CONCLUSIONES}

1). El inventario presenta validez de contenido y de constructo.

2). El inventario mide intereses para carreras técnicas con muy bajo error de medición, lo que hace del inventario un instrumento de medición psicológica confiable.

3). El sexo plantea diferencia significativa en diez de las quince escalas, razón por la cual decidimos elaborar baremos de puntaje $\mathrm{T}$, para varones y para mujeres.

\section{RECOMENDACIONES}

1). Es necesario probar la forma abreviada y correlacionar con la forma ampliada para verificar si se modifica la confiabilidad.

2). Es conveniente adaptarla y estandarizarla en el interior del país, por la importancia que tiene la orientación vocacional y profesional en la organización del plan de vida del individuo.

\section{REFERENCIAS}

Muñiz, J. (1996) Psicometría. Madrid: Ed. Universitas S.A.

Rivas Francisco (1990) Psicología vocacional: enfoques del asesoramiento. Madrid: Ed. Morata

Cassullo M. Cayssials. (1994) Proyecto de vida y decisión vocacional. B. Aires: Ed. Paidos.

OIT Informe sobre el empleo en el mundo 1998-1999. Papel fundamental de la formación.

Siegel, S. y Castellan N. (1995) Estadística no paramétrica aplicada a las ciencias de la conducta. México: Ed. Trillas.

Vicuña P. Luis. (1998) Inventario de Interés Vocacionales y Profesionales CASM 84-R98. (1984). Cedeis. Lima Perú.

Vicuña P. Luis. (2001) Concordancia entre la facultad académica a la que pertenece el alumno con los intereses vocacionales y con los estilos atribucionales de las causas que determinaron el ingreso a la UNMSM en el concurso de admisión del 2000. Revista de Investigación en Psicología. Vol 4 No. I Instituto de Investigaciones Psicológicas de la UNMSM.

Nunnally Jum. (1973) Introducción a la medición psicológica. Centro Regional de Ayuda Técnica Agencia para el Desarrollo Internacional México/Buenos Aires. Super, D. E. (1983) The history of vocational psychology: A personal perspective. En Osipow y Wallsh. Handbook o vocational psychology. Hillsdale, N.J.; LEA pp.5.38. 